\title{
Diffusion properties of electrons in GaN crystals subjected to electric and magnetic fields
}

\author{
G.I. Syngayivska*, V.V. Korotyeyev **, V.A. Kochelap \\ V. Lashkaryov Institute of Semiconductor Physics, NAS of Ukraine, 41, prospect Nauky, 03680 Kyiv, Ukraine \\ *E-mail: singg@ukr.net, \\ **E-mail: koroteev@ukr.net
}

\begin{abstract}
We have studied the diffusion coefficient of hot electrons in GaN crystals under moderate electric $(1 \ldots 10 \mathrm{kV} / \mathrm{cm})$ and magnetic $(1 \ldots 4 \mathrm{~T})$ fields. Two configurations, parallel and crossed fields, have been analyzed. The study was carried out for compensated bulklike $\mathrm{GaN}$ samples for various lattice temperatures $(30 \ldots 300 \mathrm{~K})$ and impurity concentrations $\left(10^{16} \ldots 10^{17} \mathrm{~cm}^{-3}\right)$. We found that at low lattice temperatures and low impurity concentrations, electric-field dependences of the transversal-to-current components of the diffusion tensor are non-monotonic for both configurations, while diffusion processes are mainly controlled by the magnetic field. With increasing the lattice temperature or impurity concentration, behaviour of the diffusion tensor becomes more monotonous and less affected by the magnetic field. We showed that this behaviour of the diffusion processes is caused by the distinct kinetics of hot electrons in polar semiconductors with strong electron - optical phonon coupling. We have suggested that measurements of the diffusion coefficient of electrons subjected to electric and magnetic fields facilitate identification of features of different electron transport regimes and development of more efficient devices and practical applications.
\end{abstract}

Keywords: diffusion coefficient, magneto-transport properties, hot electrons, GaN, Monte Carlo method.

doi: https://doi.org/10.15407/spqeo21.04.325

PACS 72.20.Ht, 72.20.Dp, 73.23.-b, 85.35.-p

Manuscript received 09.11.18; revised version received 27.11.18; accepted for publication 29.11.18; published online 03.12.18.

\section{Introduction}

The results of intensive investigations of wide-bandgap semiconductor compounds, in particular, group-III nitrides, such as GaN, InN, AlN and related quantum heterostructures, can find various applications in modern high-power and high-frequency microelectronics and optoelectronics [1, 2]. The nitride compounds are discussed as perspective materials for new devices like to light-emitting diodes [3, 4], optical switches [5], biosensors [6] and THz-active devices. The latter include electrically pumping $\mathrm{THz}$ sources [7-9], detectors [10] and modulators $[5,11]$. A lot of attention is also paid to the electron transport properties of the nitrides in magnetic fields to develop novel devices working as sensors and switches controlled by a magnetic field [12-14].

In contrast to conventional $\mathrm{A}^{\mathrm{III}} \mathrm{B}^{\mathrm{V}}$ materials, namely: GaAs, InSb or InP, the wide-bandgap nitrides ( $\mathrm{GaN}$ has the bandgap $3.2 \mathrm{eV}$ ) are described by large separation between the lower $\Gamma$-valley and upper valleys $(\sim 1.2 \ldots 1.5 \mathrm{eV}$ for $\mathrm{GaN})$, the high optical phonon energy, $\hbar \omega_{0}$ (for $\mathrm{GaN}, \hbar \omega_{0} \approx 92 \mathrm{meV}$ ), the strong electronoptical phonon coupling (the Fröhlich constant is $\sim 0.4$ for $\mathrm{GaN}$ ) and the high low-field mobility (at room to liquid nitrogen temperatures the mobility is $\sim 1500 \ldots 5000 \mathrm{~cm}^{2} / \mathrm{V} \cdot \mathrm{s}$ for $\left.\mathrm{GaN},[15]\right)$. These material properties of the nitrides are favorable for realization of a specific streaming-like electron transport regime, which is characterized by a quasi-periodic electron motion in the momentum space [16-20] due to the threshold character of the electron - optical phonon emission. The streaming transport regime is possible at low lattice temperatures, $T_{0},\left(k_{\mathrm{B}} T_{0}<\hbar \omega_{0}\right.$, where $k_{\mathrm{B}}$ is the Boltzmann constant) and small electron concentrations, $N_{e}$. The latter means that electron-electron scattering does not control the electron kinetics.

In the papers $[19,20]$, it was shown that conditions of the streaming transport regime can be realized in the compensated high-quality bulk GaN samples. Under streaming regime, both steady-state and high-frequency characteristics have specific behaviour. In particular, 
current-voltage characteristics show saturation, while field dependences of the diffusion coefficient demonstrate strongly non-monotonic behaviour. In addition, a high-frequency conductivity of electrons is essentially anisotropic. Spectra of the high-frequency conductivity along the steady-state field have the oscillating behavior with appearance of the frequency "windows" with negative values of the real part of the conductivity. This effect is known as the optical phonon transit-time resonance (OPTTR). The OPTTR is considered as a perspective mechanism for electricallypumped amplifiers and generators in the $\mathrm{THz}$ frequency range $[8,9]$. The streaming regime and OPTTR can be identified in the optical experiments by the methods of THz-Fourier- or time-domain spectroscopies. The peculiarities of the $\mathrm{THz}$ transmission/absorption spectra for the GaN sample under conditions of OPTTR were analyzed in [21].

Additional useful information about the streaminglike transport regime can be obtained investigating the galvano-magnetic characteristics at different configurations of the electric, $E$, and magnetic, $H$, fields. For compensated $\mathrm{GaN}$, in crossed configuration of $E$ and $H$, it was found the strong effect of the magnetic field on the streaming-like electron distribution function. In particular, it was shown that in the range of the moderate electric $(3 \ldots 10 \mathrm{kV} / \mathrm{cm})$ and magnetic $(1.5 \ldots 5 \mathrm{~T})$ fields, the electron transport occurs in the form of a vortex-like motion in the momentum space. At higher magnetic fields, the effect of a collapse of the dissipative current occurs due to the strong suppression of electron-optical phonon emission [22]. The field dependences ( $v s E$ and $H$ ) of the dissipative current, the Hall current, and the Hall electric field were studied in details for the compensated GaN [23].

This work continues investigations of the hot electron transport in compensated $\mathrm{GaN}$ with a focus on the diffusion processes of electrons in the real space. These processes are actual for systems with non-uniform electron concentrations and are described by the Fick law:

$$
J_{i}=-e D_{i j} \frac{\partial N_{e}}{\partial x_{j}},
$$

where $J_{i}$ is $i$-projection of the diffusion flux density, $D_{i j}$ is the diffusion coefficient tensor. Below we will study the $E$ - and $H$-field dependences of $D_{i j}$.

It is well-known that under equilibrium conditions ( $E=0, H=0$ ) in cubic crystals, the diffusion processes are characterized by a scalar diffusion coefficient, $D$, which obeys the Einstein relationship:

$$
D=\frac{\mu k_{\mathrm{B}} T_{0}}{e}
$$

where $\mu$ is the low-field electron mobility, and $e$ is the elementary charge. This relationship is valid for nondegenerate electrons. For the case of the weak applied electric fields, when the electron distribution function remains quasi-isotropic, the diffusion coefficient can be obtained by solving the Boltzmann transport equation and can be expressed through the integrals of a steadystate distribution function in the momentum space [24, 25]. Under strongly non-equilibrium conditions, two approaches for calculations of the diffusion coefficient are used. Both are based on the Monte Carlo simulation of the electron transport. The first approach is based on calculations of the variance of random distances of travels of individual electron per unit time [26, 27]. The obtained diffusion coefficient describes the spreading of initially localized in space electron packet, i.e., it directly corresponds to the Fick law. The second approach uses computation of the velocity autocorrelation function in the time domain $[27,28]$. Both approaches give the same results in the case of the absence of the electron-electron correlation [29].

The experimental techniques of the diffusion coefficient measurements are based on the electrogradient measurements of the thermo-electric force [30], time-of-flight measurements [31], noise spectroscopy [32], light-induced grating technique [33, 34], etc.

The mentioned above theoretical and experimental studies of diffusion properties of the non-equilibrium electron gas are dated by 1970-1980-th and concentrated to conventional semiconductors such as $\mathrm{Si}, \mathrm{Ge}, \mathrm{GaAs}$, etc. In the past decades, the theoretical studies of the diffusion properties of hot electrons were oriented on the nitride compounds, GaN [35] and InN [36]. Particularly, in these papers, the electric field dependences of the diffusion coefficient were obtained for the wide range of electric fields: $E=0 \ldots 100 \mathrm{kV} / \mathrm{cm}$. The non-monotonic behaviour of the diffusion coefficient was identified for both longitudinal and transversal diffusion with respect to the electric field direction.

The present interest to the study of the diffusion processes in a non-equilibrium electron gas is inspired, in the particular, by the development of recent pure alloptical pump-probe techniques, known as the lightinduced transient grating (LITG) technique [37]. This contactless technique allows determining the diffusion coefficient as well as the carrier recombination times by investigating temporal changes of the diffraction efficiency of the grating induced optically near the surface of material.

The aim of this paper is to theoretically study the diffusion processes of hot electrons in compensated $\mathrm{GaN}$ subjected to the electric and magnetic fields of moderate magnitudes. The paper is organized as follows. In Section II, we briefly describe the model of the electron transport and the Monte Carlo method used for determination of the diffusion tensor. In Section III, we discuss the steady-state electric characteristics for different samples of $\mathrm{GaN}$ at zero magnetic field. Then, the effect of the magnetic field on the diffusion coefficient is considered for the parallel and crossed configurations of electric and magnetic fields in Sections IV and V, respectively. In Section VI, we summarize the main results of these investigations. 


\section{The model of the electron transport in electric and magnetic fields}

We consider the electron transport in the samples of GaN of cubic modification under the parallel and crossed configurations of electric, $E$, and magnetic, $H$, fields. At $E \| H$, we assume that $E$ and $H$ are directed along $z$-axis (Fig. 1a). In the case $E \perp H$, it is supposed that $E$ and $H$ are applied along $z$ - and $y$-axis, respectively (Fig. 1b). Here, the GaN-samples are assumed to be with shortcircuited Hall contacts.

To calculate electron transport characteristics, we used the single-particle algorithm of the Monte Carlo procedure $[20,22,23,38]$. Three main scattering mechanisms were taken into account: electron scattering by ionized impurities, acoustic phonons and polar optical phonons. We considered the range of the electric fields for which the intervalley transitions to the upper valleys are absent and the electron dispersion law can be set the parabolic one. The electron-electron scattering was not included into these calculations, because only small electron concentrations were considered.

The standard single-particle Monte Carlo algorithm is based on the stochastic simulation of the electron trajectories in the momentum and coordinate spaces including the scattering processes (for details, see [39, 40]). The instantaneous values of the electron velocity (momentum and/or energy) and coordinate are recorded at the specific moments of time and accumulated for the further statistical processing. Motion of electrons in the electric and magnetic fields were treated as semiclassical. These fields were taken into account when modelling the electron trajectory between sequential collisions; however, the fields were not included to the scattering probabilities.

In the case of parallel configuration for $E$ and $H$, the equations of the electron motion in momentum space are as follows:

$$
\left\{\begin{array}{l}
\dot{p}_{x}=\omega_{c} p_{y} \\
\dot{p}_{y}=-\omega_{c} p_{x} \\
\dot{p}_{z}=e E
\end{array}\right.
$$

For the crossed configuration, they are read as:

$$
\left\{\begin{array}{l}
\dot{p}_{x}=-\omega_{c} p_{z} \\
\dot{p}_{y}=0 \\
\dot{p}_{z}=e E+\omega_{c} p_{x}
\end{array}\right.
$$

Here, $p_{x}, p_{y}, p_{z}$ are the components of the electron momentum, $\omega_{c}$ is the cyclotron frequency, $\omega_{c}=e H / m^{*} c$, $m^{*}$ and $c$ are the effective electron mass and light velocity, respectively.
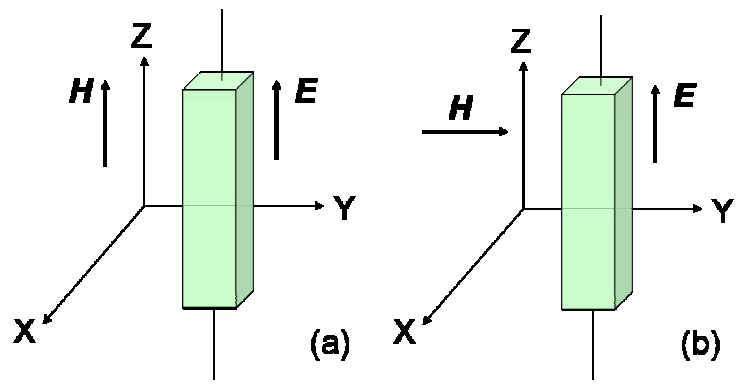

Fig. 1. The scheme of parallel (a) and crossed configurations of $E$ and $H$.

At the end of a free flight, the electron velocity is calculated by the following equations for parallel

$\left\{\begin{array}{l}p_{x}(t)=p_{x}\left(t_{0}\right) \cos \left[\omega_{c}\left(t-t_{0}\right)\right]+p_{y}\left(t_{0}\right) \sin \left[\omega_{c}\left(t-t_{0}\right)\right] \\ p_{y}(t)=-p_{x}\left(t_{0}\right) \sin \left[\omega_{c}\left(t-t_{0}\right)\right]+p_{y}\left(t_{0}\right) \cos \left[\omega_{c}\left(t-t_{0}\right)\right] \\ p_{z}(t)=p_{z}\left(t_{0}\right)+e E\left(t-t_{0}\right)\end{array}\right.$

and crossed configurations

$$
\left\{\begin{aligned}
p_{x}(t)= & \left(p_{x}\left(t_{0}\right)+e E / \omega_{c}\right) \cos \left[\omega_{c}\left(t-t_{0}\right)\right]- \\
& -p_{z}\left(t_{0}\right) \sin \left[\omega_{c}\left(t-t_{0}\right)\right]-e E / \omega_{c} \\
p_{y}(t)= & p_{y}\left(t_{0}\right) \\
p_{z}(t)= & \left(p_{x}\left(t_{0}\right)+e E / \omega_{c}\right) \sin \left[\omega_{c}\left(t-t_{0}\right)\right]+ \\
& +p_{z}\left(t_{0}\right) \cos \left[\omega_{c}\left(t-t_{0}\right)\right]
\end{aligned}\right.
$$

Here, $t_{0}$ and $t$ are, respectively, initial and final moments of a free flight.

The calculations of the electron coordinates are easily incorporated into the Monte Carlo scheme. The following equations are used in our Monte Carlo procedure for simulation of the electron trajectory in the coordinate space:

$$
\left\{\begin{aligned}
x(t)= & x\left(t_{0}\right)+\frac{p_{y}\left(t_{0}\right)}{m^{*} \omega_{c}}+\frac{p_{x}\left(t_{0}\right)}{m^{*} \omega_{c}} \sin \left[\omega_{c}\left(t-t_{0}\right)\right]- \\
& -\frac{p_{y}\left(t_{0}\right)}{m^{*} \omega_{c}} \cos \left[\omega_{c}\left(t-t_{0}\right)\right] \\
y(t)= & y\left(t_{0}\right)-\frac{p_{x}\left(t_{0}\right)}{m^{*} \omega_{c}}+\frac{p_{x}\left(t_{0}\right)}{m^{*} \omega_{c}} \cos \left[\omega_{c}\left(t-t_{0}\right)\right]+ \\
& +\frac{p_{y}\left(t_{0}\right)}{m^{*} \omega_{c}} \sin \left[\omega_{c}\left(t-t_{0}\right)\right] \\
z(t)= & z\left(t_{0}\right)+p_{z}\left(t_{0}\right)\left(t-t_{0}\right) / m^{*}+ \\
& +e E\left(t-t_{0}\right)^{2} / 2 m^{*}
\end{aligned}\right.
$$




$$
\left\{\begin{array}{c}
x(t)=x\left(t_{0}\right)+\frac{p_{x}\left(t_{0}\right)+e E / \omega_{c}}{m^{*} \omega_{c}} \sin \left[\omega_{c}\left(t-t_{0}\right)\right]-\frac{p_{z}\left(t_{0}\right)}{m^{*} \omega_{c}}+ \\
+\frac{p_{z}\left(t_{0}\right)}{m^{*} \omega_{c}} \cos \left[\omega_{c}\left(t-t_{0}\right)\right]-\frac{e E}{m^{*} \omega_{c}}\left(t-t_{0}\right) \\
y(t)=y\left(t_{0}\right)+p_{y}\left(t_{0}\right)\left(t-t_{0}\right) / m^{*} \\
z(t)=z\left(t_{0}\right)-\frac{p_{x}\left(t_{0}\right)+e E / \omega_{c}}{m^{*} \omega_{c}} \cos \left[\omega_{c}\left(t-t_{0}\right)\right]+\frac{p_{x}\left(t_{0}\right)}{m^{*} \omega_{c}}+ \\
+\frac{p_{z}\left(t_{0}\right)}{m^{*} \omega_{c}} \sin \left[\omega_{c}\left(t-t_{0}\right)\right]+\frac{e E}{m^{*} \omega_{c}^{2}}
\end{array}\right.
$$

The equations (5a) and (5b) were used for determination of electron coordinates in the case of the parallel and crossed configurations, respectively.

To calculate the components diffusion tensor, $D_{i j}$, we used the following equation [27, 39, 40]:

$$
D_{i j}=\frac{1}{2} \frac{d}{d t}\left\langle\left(r_{i}(t)-\left\langle r_{i}(t)\right\rangle\right)\left(r_{j}(t)-\left\langle r_{j}(t)\right\rangle\right)\right\rangle,
$$

where $r_{i, j=1,2,3}=\{x, y, z\}$ were calculated according to equations $(5 a)$ or $(5 b)$. Angle brackets denote the time average.

In the zeroth magnetic field and for the parallel configuration of the fields, $E \| H$, the tensor of the diffusion coefficients has three non-zero diagonal components: $D_{x x}, D_{y y}, D_{z z}$ and $D_{x x}=D_{y y}$. (see $E-H$ orientation in Fig. 1). At $E \perp H$, the tensor $D_{i j}$ have five non-zero components $D_{x x}, D_{y y}, D_{z z}, D_{x z}$ and $D_{z x}=D_{x z}$. Below, we will analyze the components of the tensor $D_{i j}$ corresponding to the directions transversal to the electric field.

\section{Electrical characteristics at $\boldsymbol{H}=\mathbf{0}$}

In this section we discuss the electron transport characteristics including the drift velocity, $V_{d}$, the average energy, $\langle\varepsilon\rangle$, as well as the diagonal components, $D_{x x}=D_{y y}$, of the diffusion tensor, all as functions of the electric field at the absence of the magnetic field. The parameters $D_{x x}$ and $D_{y y}$ describe electron diffusion transversal to the applied field. We present the electric characteristics for three particular examples, which differ by the lattice temperature, $T_{0}$, the concentration of ionized impurities, $N_{i}$, and the electron concentration, $N_{e}$. For the case I, we assume the following parameters: $N_{i}=10^{16} \mathrm{~cm}^{-3}, N_{e}=10^{15} \mathrm{~cm}^{-3}$ and $T_{0}=30 \mathrm{~K}$, for the case II $-N_{i}=10^{17} \mathrm{~cm}^{-3}, N_{e}=10^{16} \mathrm{~cm}^{-3}$ and $T_{0}=30 \mathrm{~K}$, and for the case III $-N_{i}=10^{16} \mathrm{~cm}^{-3} N_{e}=10^{15} \mathrm{~cm}^{-3}$ and $T_{0}=300 \mathrm{~K}$. The features of the streaming transport regime are expected to be well-pronounced for the case I.

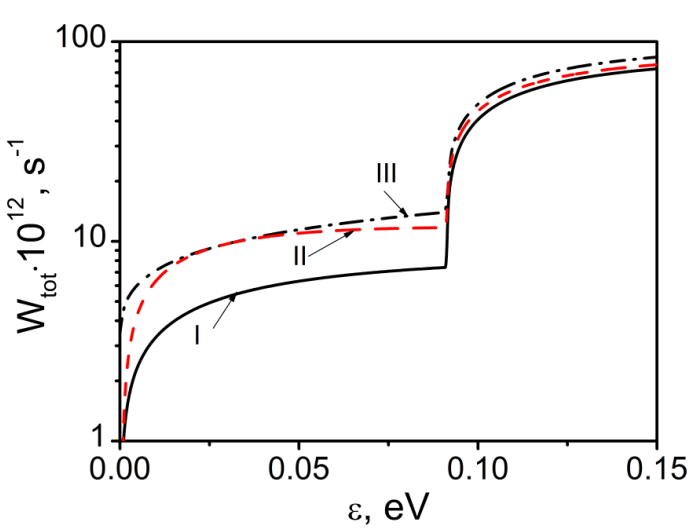

Fig. 2. Scattering probability $W_{\text {tot }}$ vs the electron energy $\varepsilon$. Calculations are performed for three cases described in the text: case I - solid curve, II - dashed curve, III - dash-dotted curve.

Fig. 2 demonstrates the total scattering probability, $W_{\text {tot }}(\varepsilon)$, as a function of the electron energy, $\varepsilon$, calculated for three discussed cases. The curves marked by I, II and III correspond to the cases I, II and III, respectively. The larger difference between values of $W_{t o t}$ in the passive $\left(\varepsilon<\hbar \omega_{0}\right)$ and active $\left(\varepsilon>\hbar \omega_{0}\right)$ energy regions is preferable to realize the well-developed streaming transport regime (the case I). The large values of the total scattering probability in the active region correspond to the intensive optical phonon emission. These values are two orders larger than those in the passive region, where the less intensive acoustic phonon and ionized impurity scatterings occur at $T_{0}=30 \mathrm{~K}$. Increasing the impurity concentration (the case II) at a given temperature leads to increasing $W_{\text {tot }}(\varepsilon)$ in the passive region due to the increase of the electron-impurity scattering. The increase of $W_{\text {tot }}(\varepsilon)$ in the passive region with increasing the temperature (the case III) at a given impurity concentration is associated with activation of absorption by optical phonons.

The field dependences of the drift velocity, $V_{d}(E)$, the average electron energy, $\langle\varepsilon\rangle$, the average energy correspondent to the transversal electron motion, $\left\langle\varepsilon_{\perp}\right\rangle=\left(p_{x}^{2}+p_{y}^{2}\right) / 2 m^{*}$, and the transversal component of the diffusion coefficient, $D_{x x}(E)$, are shown in Fig. 3. As expected, the characteristic features of the streaming regime are observed only for the sample I. In the range of $E=3 \ldots 10 \mathrm{kV} / \mathrm{cm}$, the drift velocity and the average energy saturate (see Figs. $3 a$ and $3 b$ ) reach one half of the characteristic velocity $V_{0}=\sqrt{2 \hbar \omega_{0} / m^{*}}$, and the average energy approaches to $\hbar \omega_{0} / 3$, respectively. As seen, for the case II with larger impurity concentration, a welldeveloped streaming regime is not formed. At room temperature, in the range of $E=3 \ldots 10 \mathrm{kV} / \mathrm{cm}$, electron gas remains almost quasi-equilibrium, so $V_{d}(E)$ shows linear behaviour, and $\langle\varepsilon\rangle(E)$ is close to its equilibrium value of $3 k_{\mathrm{B}} T_{0} / 2$. 

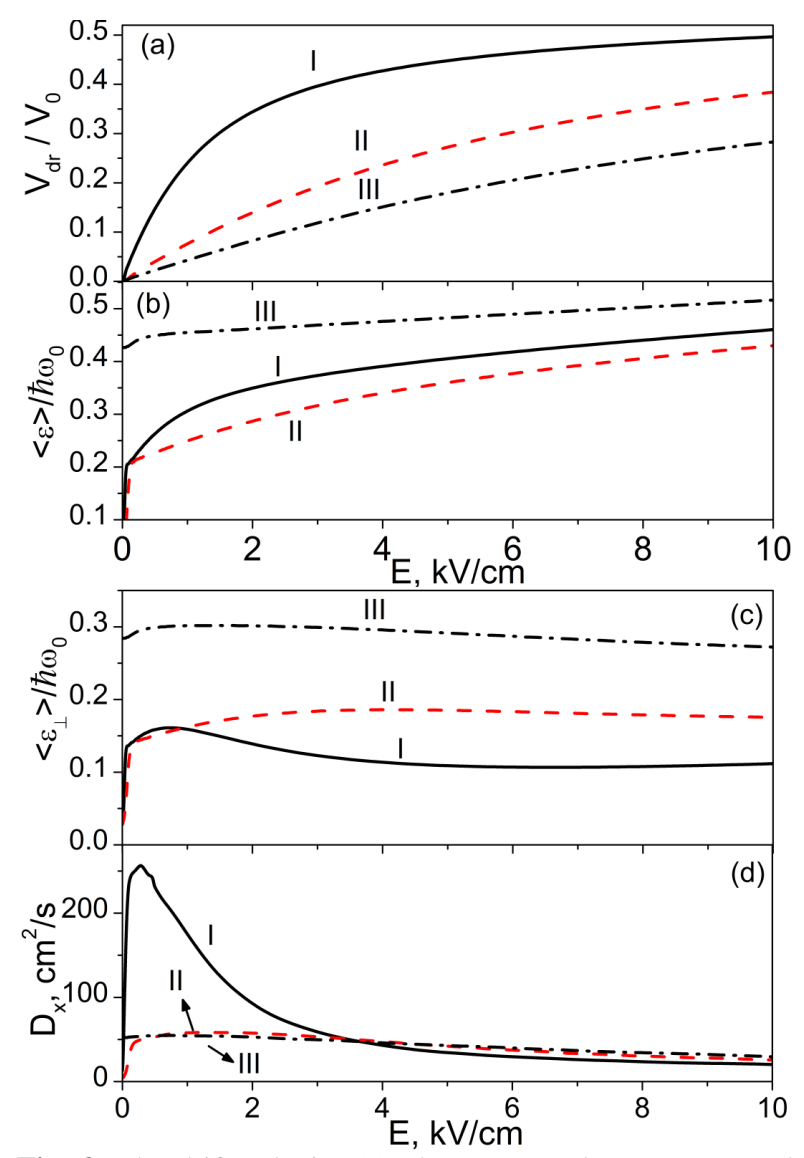

Fig. 3. The drift velocity (a), the average electron energy (b), the transverse component of the average energy (c) and the diffusion coefficient (d) vs $E$ at $H=0$ for three cases (curves I, II, III) discussed in the text. $V_{0}=4 \cdot 10^{7} \mathrm{~cm} / \mathrm{s}$ and $\hbar \omega_{0} \approx 92 \mathrm{meV}$.

The emergence of streaming regime can be clearly identified by the strong non-monotonic field dependence of the transversal component of the average electron energy, $\left\langle\varepsilon_{\perp}\right\rangle$ (see Fig. 3c). This dependence is observed for the case I. The increase of $\langle\varepsilon\rangle$ at $E$ up to $\sim 1 \mathrm{kV} / \mathrm{cm}$ is associated with initial heating the electron gas. With further increasing the field, $\left\langle\varepsilon_{\perp}\right\rangle$ decreases due to formation of a streaming-like distribution function elongated along the field direction [20]. This decrease tends to saturation in the field range $3 \ldots 10 \mathrm{kV}$. For the cases II and III, the streaming is not formed, and $\langle\varepsilon\rangle$ has a slightly non-monotonic character.

The field dependences of the transversal-to- $E$ component of the diffusion coefficient, $D_{x x}(E)$, (shown in Fig. 3d) are qualitatively similar to the dependences of $\langle\varepsilon\rangle$. The specific strong non-monotonic dependence of $D_{x x}(E)$ is inherent to the streaming regime, that is realized for the case I. At $E<500 \mathrm{~V}$, the magnitude of $D_{x x}$ rapidly increases from the equilibrium value of $13 \mathrm{~cm}^{2} / \mathrm{s}$ to the maximum of about $250 \mathrm{~cm}^{2} / \mathrm{s}$. The isotropic spreading in the transversal direction of electrons in the momentum space is the main reason for this growth of the diffusion coefficient. The maximum of $D_{x x}$ corresponds to the electric field, at which the rapid spreading of the distribution function is terminated. In this field, the essential part of high energy electrons loses its energy due to emission of optical phonons. With further increasing $E$, the streaming-like distribution function begins to form. In this case, $D_{x x}$ rapidly decreases and at $E>5 \mathrm{kV} / \mathrm{cm}$ approaches to values $20 . .25 \mathrm{~cm}^{2} / \mathrm{s}$. For the cases II and III, $D_{x x}(E)$ slowly decreasing from 50 down to $30 \mathrm{~cm}^{2} / \mathrm{s}$ with increasing $E$ from 1 up to $10 \mathrm{kV} / \mathrm{cm}$.

The effect of the magnetic field on behaviour of the diffusion coefficient is analyzed in the following sections.

\section{Diffusion coefficient at the $E \| H$ configuration}

It should be noted that for electrons with parabolic dispersion law, application of the magnetic field along the electric one has no effect on transport characteristics $V_{d}(E)$ and $\left\langle\varepsilon_{\perp}\right\rangle(E)$ [38]. It follows from the equations (3a), (4a), where it is seen that electron motion in directions along and transverse to fields is uncoupled. However, the electron diffusion process in the coordinate space shows the strong dependence on the magnetic field, $H$.

Fig. 4 demonstrates the electric field dependences of the transversal-to-current component of the diffusion tensor, $D_{x x}(E)$, calculated for three values of $H$. Panels (a), (b) and (c) correspond to the cases I, II and III, respectively. For comparison, the component $D_{x x}(E)$ at

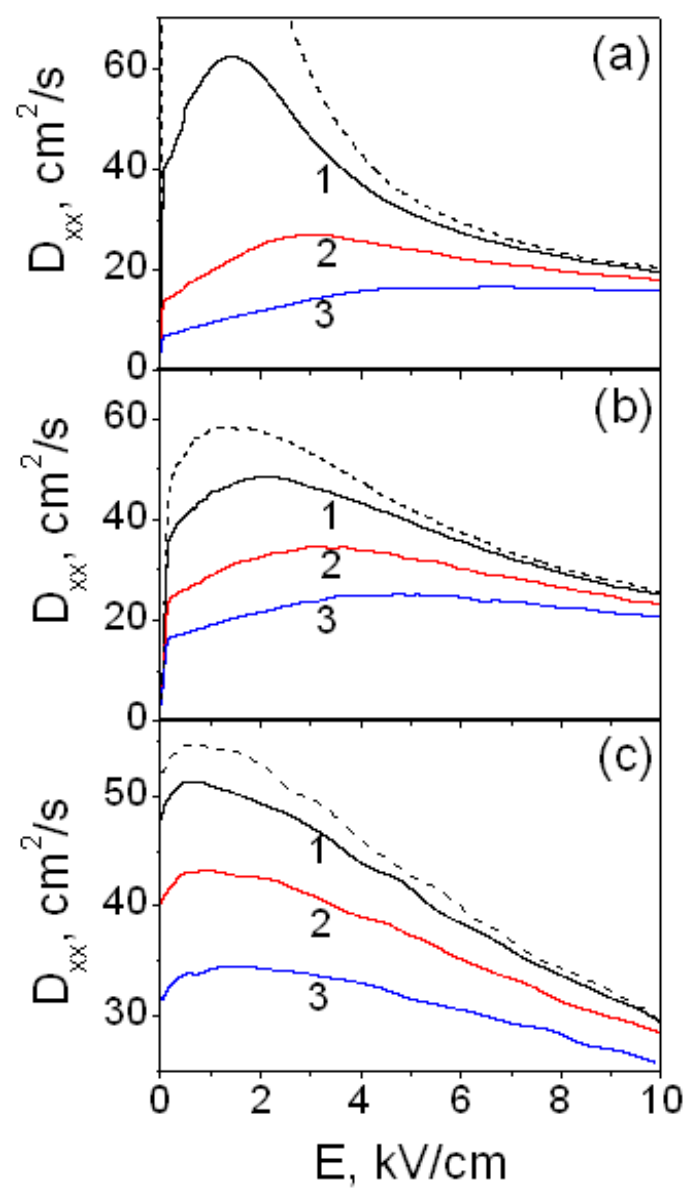

Fig. 4. Dependences of $D_{x x}(E)$ at $E \| H$ for the case I - (a); II (b); III - (c) at $H=0$ (dashed curves), $H=1.1 \mathrm{~T}$ (curves 1 ), $2.3 \mathrm{~T}$ (curves 2), $3.4 \mathrm{~T}$ (curves 3). 


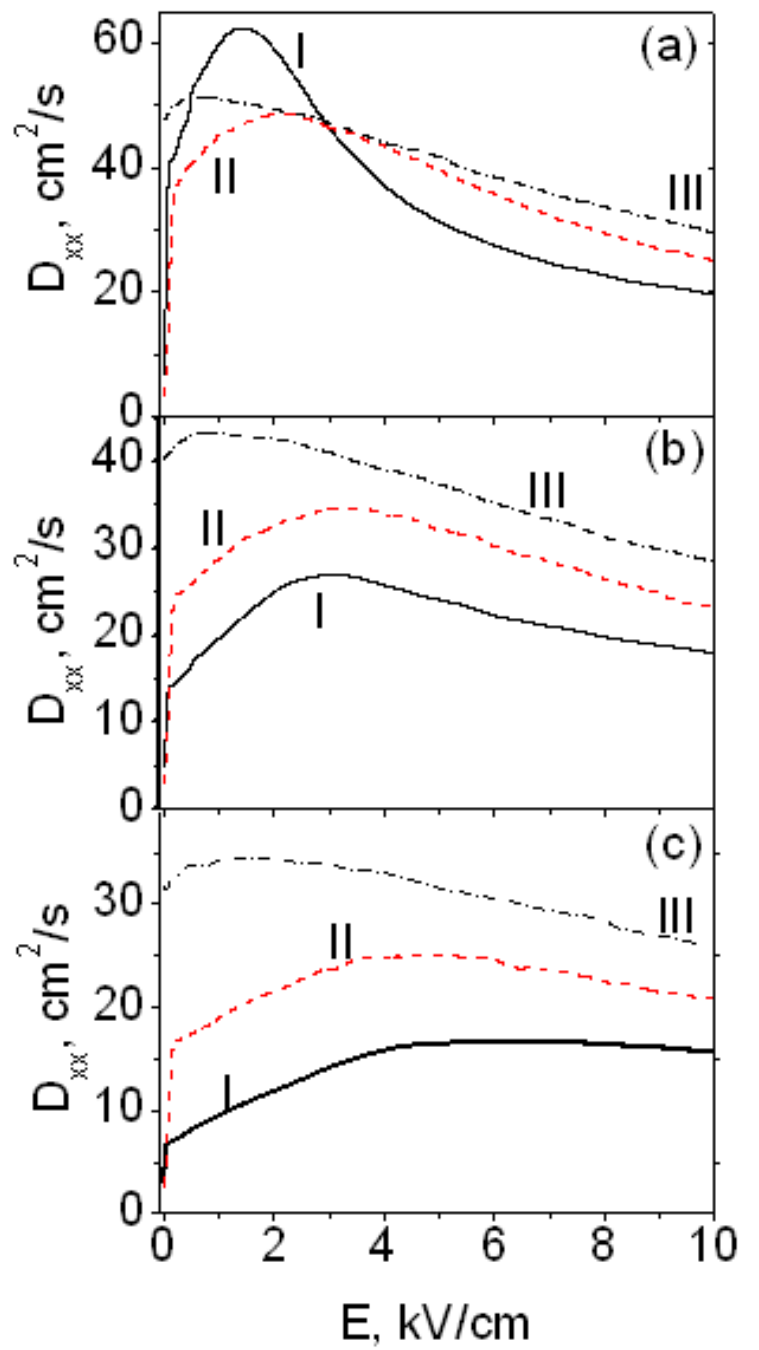

Fig. 5. Dependences $D_{x x}(E)$ at the given values of $H=1.1 \mathrm{~T}$ (a), $2.3 \mathrm{~T}$ (b), $3.4 \mathrm{~T}$ (c). Curves I, II and III correspond to the case I, II and III, respectively.

$H=0$ is shown by the dashed curve. The curves 1,2 and 3 correspond to three magnitudes of the magnetic field of $1.1,2.3$ and $3.4 \mathrm{~T}$, respectively. Behaviour of the dependence $D_{x x}(E)$ in magnetic field has the following general peculiarities for all the cases: (i) the dependences of $D_{x x}(E)$ are non-monotonic with their maximum shifted to the higher electric fields with increasing $H$; (ii) the magnetic field suppresses diffusion in transversal directions with respect to $E$ and $H$; (iii) the effect of magnetic field decreases at higher electric fields.

The magnetic field effect on the electron diffusion is more essential for the case I, for which the streaming regime is realized. Even at weak magnetic fields, it is observed strong suppression of the maximum of diffusion coefficient, which decreases from the value $\sim 250 \mathrm{~cm}^{2} / \mathrm{s}$ at $H=0$ down to $\sim 60 \mathrm{~cm}^{2} / \mathrm{s}$ at $H=1.1 \mathrm{~T}$. With further increasing the magnetic field, the maximum of $D_{x x}$ progressively decreases and has the values close to $30 \mathrm{~cm}^{2} / \mathrm{s}$ at $H=2.3 \mathrm{~T}$ and approximately $15 \mathrm{~cm}^{2} / \mathrm{s}$ at $H=3.4 \mathrm{~T}$. The position of maximum of the diffusion coefficient correspond to the electric fields of $0.5,1.5,3$ and $5.5 \mathrm{kV} / \mathrm{cm}$ for $H=0,1.1,2.3$ and $3.4 \mathrm{~T}$, respectively.
For the cases II and III, for which the streaming regime does not occurs, the magnetic field more weakly modifies the diffusion coefficient. For example, the maximum of $D_{x x}$ decreases only twice from $\sim 60 \mathrm{~cm}^{2} / \mathrm{s}$ at $H=0$ down to $\sim 30 \mathrm{~cm}^{2} / \mathrm{s}$ at $H=3.4 \mathrm{~T}$.

Fig. 5 allows us to compare the diffusion coefficient for these three cases at given magnetic fields. For the weak magnetic field $(H=1.1 \mathrm{~T})$, there is the range of the electric fields $E=0 . \ldots 3 \mathrm{kV} / \mathrm{cm}$, where the diffusion coefficient for case I is higher than that for the cases II and III (see the panel (a) in Fig. 5). At larger $H, D_{x x}$ for the case I is lower than $D_{x x}$ for the cases II and III in the studied electric fields (see the panels (b) and (c) in Fig. 5).

The results of our modelling the diffusion processes in compensated $\mathrm{GaN}$ show that the largest variations of $D_{x x}(E)$ with the magnetic field occur in the samples, for which the streaming electron transport is formed. Both the electro-gradient and optical measurements (using LITG techniques) of the diffusion coefficient can provide additional experimental tools for identification of the streaming transport regime and hot electron parameters for GaN crystals.

\section{Diffusion coefficient at the $E \perp H$ configuration}

In this section, we consider the field dependences of components of the diffusion tensor corresponding to the transversal motion with respect to the electric field directions. In the case $E \perp H$, assuming that $E$ and $H$ are oriented along $z$-axis and $y$-axis, respectively (see Fig. 2b), two components of the diffusion tensor, $D_{x x}$ and $D_{y y}$, will be analyzed. The $D_{x x}$ component describes the diffusion current perpendicular to $E$ and $H$. The $D_{y y}$ component characterizes the diffusion in the direction parallel to $H$ and perpendicular to $E$.

As discussed previously [22, 23, 41, 42], the magnetic field for this configuration strongly affects on the transport characteristics. It was established that the streaming transport regime can be destroyed by the magnetic field, forming a vortex-like distribution function in the momentum space. The result of the measurements of current-voltage characteristics depends on the form of the external circuits. Our transport model assumes the short-circuited Hall contacts.

In the cases $E \perp H$ as well as $E \| H$, behavior of $D_{x x}(E)$ depends on material parameters of the cases and the relation between magnitudes of $E$ and $H$. The behavior of the component $D_{x x}$ has the same general features (i)-(iii), which are listed in previous section. As well seen from Fig. 6a, the impact of the magnetic field on the diffusion is largest for the case I. The dependences of $D_{x x}(E)$ show non-monotonic behavior with a maximum shifted to the region of higher electric fields with increasing $H$. However, at a given non-zero $H$, there is a region of electric fields, where diffusion is more intense than at $H=0$. This peculiarity was absent in the case of $E \| H$. Moreover, the maximum of $D_{x x}(E)$ is more essentially shifted for the configuration $E \perp H$. For example, at $H=1.1 \mathrm{~T}$, the maximum of $D_{x x}$ is realized at 


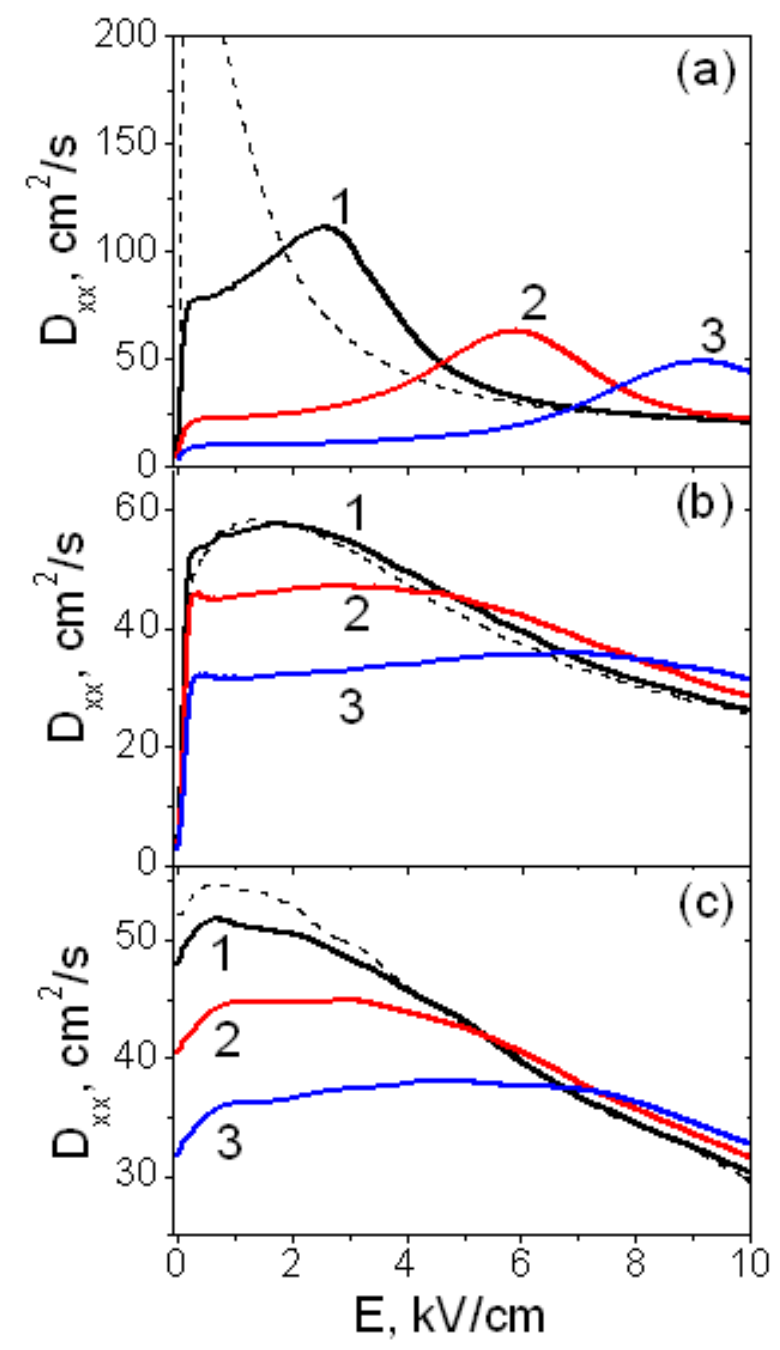

Fig. 6. Dependences $D_{x x}(E)$ at $E \perp H$ for the case I - (a), II - (b), III - (c) at $H=0$ (dashed curves), $H=1.1 \mathrm{~T}$ (curves 1 ), $2.3 \mathrm{~T}$ (curves 2), $3.4 \mathrm{~T}$ (curves 3).

$E=3 \mathrm{kV} / \mathrm{cm}$ (for comparison, in parallel configuration at $E=1.5 \mathrm{kV} / \mathrm{cm}$ ). For the cases II and III (panels (b) and (c) in Fig. 6, respectively), the effect of the magnetic field is weak, the dependences of $D_{x x}(E)$ are weakly nonmonotonous with an extended region of the electric field $E=1 \ldots 6 \mathrm{kV} / \mathrm{cm}($ at $H=2.3 \ldots 3.4 \mathrm{~T})$, where $D_{x x}(E)$ is the practically constant.

In Fig. 7, the same dependences of $D_{x x}(E)$ are grouped accordingly to the given magnetic fields. As seen, at $H=1.1 \mathrm{~T}$ the $D_{x x}$ component calculated for the case I exceeds the values of $D_{x x}$ obtained for the cases II and III within the range $E=0.1 \ldots 5 \mathrm{kV} / \mathrm{cm}$. With increasing $H$, this range decreases and shifts to the higher electric fields. For the cases II and III, the field dependences of $D_{x x}$ are very similar in wide ranges of $E$ and $H$.

The well-pronounced maximum of the diffusion tensor $D_{x x}$ component is realized for the case I. It should be noted that the maximum arises in such fields $E$ and $H$ at which formation of the specific form of steady-state distribution function in the momentum space

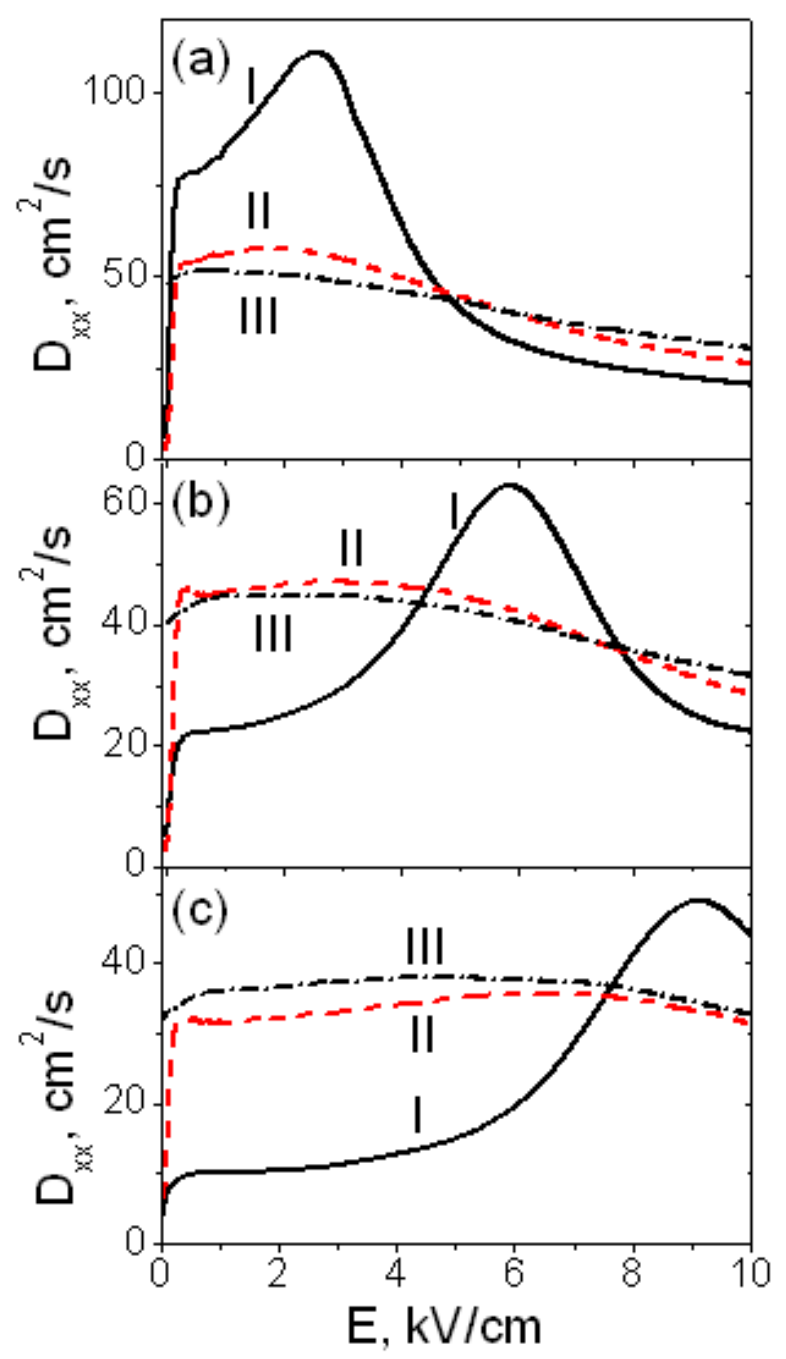

Fig. 7. Dependences $D_{x x}(E)$ at the given values of $H=1.1 \mathrm{~T}$ (a), $2.3 \mathrm{~T}(\mathrm{~b}), 3.4 \mathrm{~T}$ (c). Curves I, II and III correspond to the case I, II and III, respectively.

occurs. This distribution function describes the magnetotransport regime with co-existence of two separated electron groups (for details, see [22, 42]).

In contrast to the parallel configuration of $E$ and $H$, in the crossed configuration components $D_{x x}$ and $D_{y y}$ are not equal. $D_{y y}$ component describes the diffusion current along the magnetic field. The electric field dependences of $D_{y y}$ at several values of $H$ are shown in Fig. 8. As seen, behavior of $D_{y y}(E)$ cardinally differs from $D_{x x}(E)$. In general, the increase of $H$ leads to the growth of electron diffusion in the $y$-direction for a wide range of the electric fields. Particularly, for the case I (see panel (a) in Fig. 8) the amplitude and position of the maximum are weakly modified with increasing $H$ within the range 1.1...3.4 T. However, at weak magnetic fields, $0 \ldots 1.1 \mathrm{~T}$, the amplitude of $D_{y y}$ maximum is twice increased. With increasing the electric field, diffusion along the direction of magnetic field progressively decreases, and at $E>6 \mathrm{kV} / \mathrm{cm}$ it tends to the case corresponding to $H=0$. For the case II, the impact of the magnetic field on the dependence $D_{y y}(E)$ is not so essential as for the case I. 


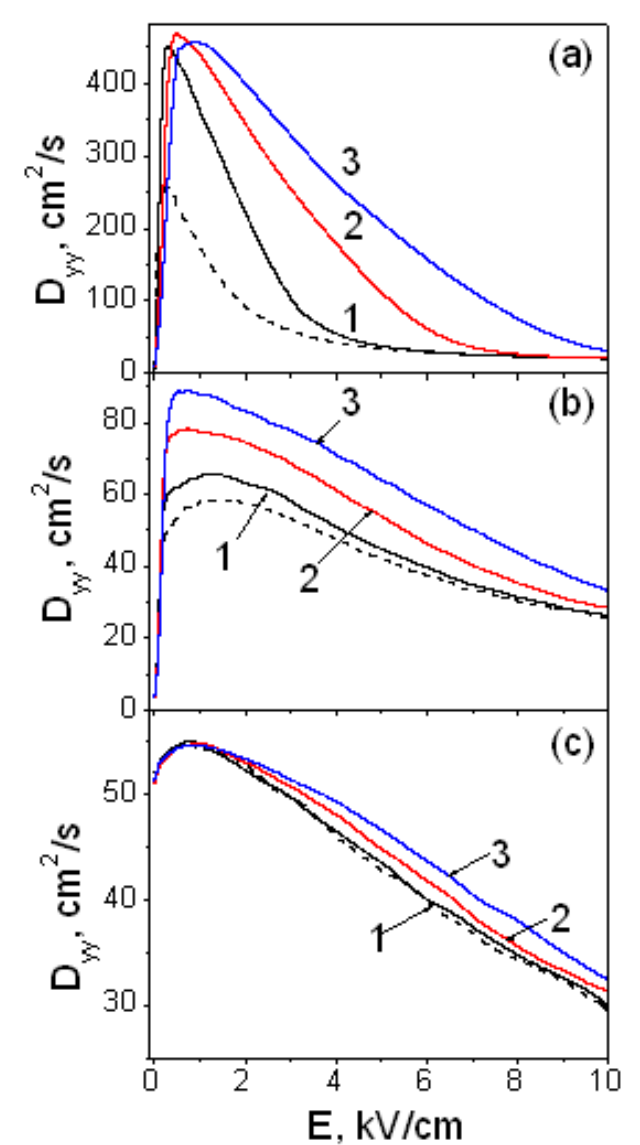

Fig. 8. Dependences $D_{y y}(E)$ at $E \perp H$ for the case I - (a); II - (b); III - (c) at $H=0$ (dashed curves), $H=1.1 \mathrm{~T}$ (curves 1 ), $2.3 \mathrm{~T}$ (curves 2), $3.4 \mathrm{~T}$ (curves 3).

The maximum of $D_{y y}$ is increased by $\sim 30 \%$ with increasing the magnetic field from 0 up to $3.4 \mathrm{~T}$. For the case III, the magnetic field does not influence on the dependence $D_{y y}(E)$.

We found that general behaviour of galvanomagnetic properties of $D_{y y}$ component in the diffusion tensor correlates with behavior of the average energy of the electron motion in $y$-direction, $\left\langle\varepsilon_{y}\right\rangle$. The results of our calculations of the electric field dependences, $\left\langle\varepsilon_{y}\right\rangle$, for the three samples are shown in Fig. 9 for three values of $H$.

\section{Conclusions}

We have studied the diffusion properties of hot electrons in bulk-like GaN samples for parallel and crossed configurations of the applied electric and magnetic fields. It has been analyzed the field dependences of the transversal components in the diffusion tensor, which correspond to electron motion in the directions perpendicular to the electric field. These results have been obtained by the Monte Carlo method of calculations. It was analyzed three types of $\mathrm{GaN}$ samples with the degree of compensation close to $90 \%$. It was assumed $T_{0}=30 \mathrm{~K}, N_{i}=10^{16} \mathrm{~cm}^{-3}$ for the sample I, $T_{0}=30 \mathrm{~K}, N_{i}=10^{17} \mathrm{~cm}^{-3}$ for the sample II, and $T_{0}=300 \mathrm{~K}, N_{i}=10^{16} \mathrm{~cm}^{-3}$ for the sample III). Parameters

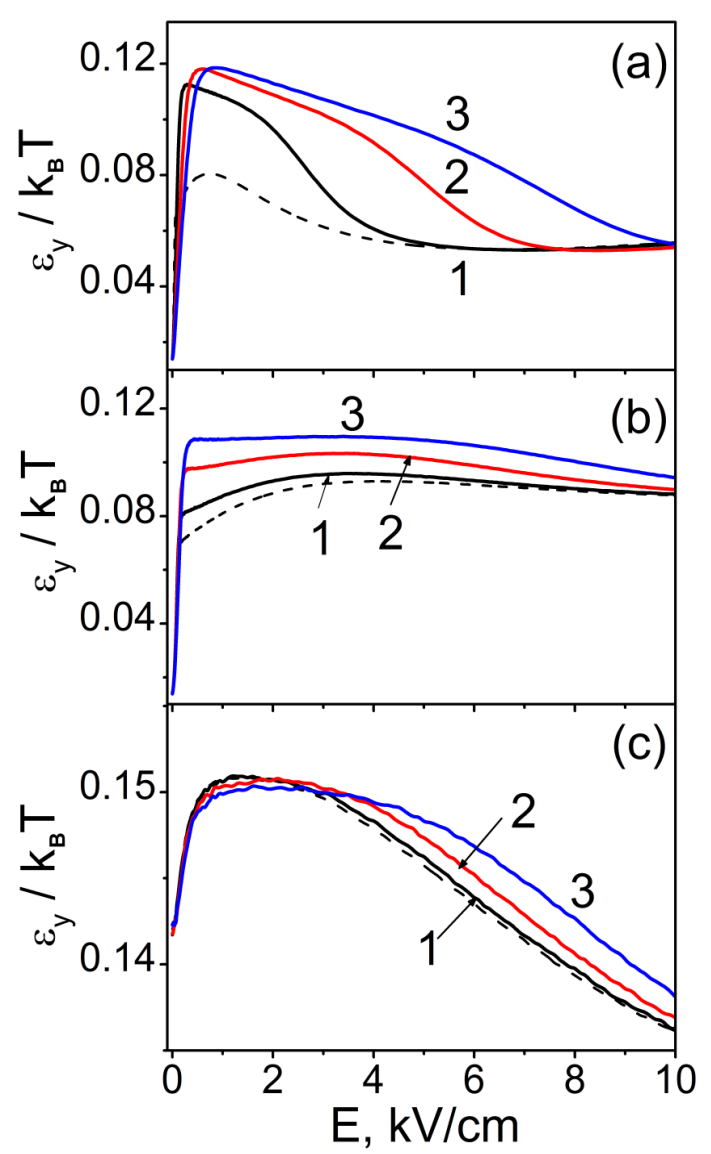

Fig. 9. Dependences $\left\langle\varepsilon_{y}\right\rangle(E)$ for the case I - (a), II - (b), III - (c) at $H=0$ (dashed curves), $H=1.1 \mathrm{~T}$ (curves 1 ), $2.3 \mathrm{~T}$ (curves 2 ), $3.4 \mathrm{~T}$ (curves 3 ).

of the sample I satisfy the requirements for realizing the streaming transport regime.

We found that a strong impact of magnetic fields on diffusion properties of the electron gas takes place for the sample I. In the parallel configuration of $E$ and $H$, the electric field dependences of the transversal-to-current components of the diffusion tensor have non-monotonous behavior with a maximum. The amplitude and position of the maxima depend on the magnitudes of magnetic field and are changed with the increase of magnetic field: the maximum is decreased, and its position is shifted to the higher electric fields.

In the crossed configuration of $E$ and $H$, the transversal-to-fields component of the diffusion tensor has similar behaviour. However, the positions of the maximum are more sensitive to $H$ variation.

The suppression of electron diffusion in transversal to $H$ direction with the increase of magnetic field is a general phenomenon that is observed in both configurations. However, in the crossed configuration, the magnetic field enhances electron diffusion along $H$ direction. The electric field dependences of the longitudinal-to- $H$ component of the diffusion tensor also have the maximum. Both position and amplitude of the maximum weakly depend on the magnitudes of $H$. 
The parameters of the samples II and III prevent formation of the streaming transport regime. This is the main reason of the weak influence of electric and magnetic fields on diffusion properties of electrons in these samples. However, the main peculiarities observed in the field dependences of diffusion processes for the sample I take place for the samples II and III as well.

We suggest that the streaming transport regime and related magneto-transport effects can be investigated by measurements of the diffusion effects of hot electrons in the electric and magnetic fields. Both the electro-gradient and optical measurements (using LITG techniques) of the diffusion coefficient can be used for this purpose. Also, the knowledge of high-field dependences of the diffusion coefficient is important for modeling the micron-scale electronic devices operating in strong electric and magnetic fields.

\section{Acknowledgments}

This work is partially supported by the Ministry of Education and Science of Ukraine (Project M/24-2018) and German Federal Ministry of Education and Research (BMBF Project 01DK17028).

\section{References}

1. Millan J., Godignon P., Perpina X., Perez-Tomas A. and Rebollo J. A survey of wide bandgap power semiconductor devices. IEEE Trans. Power Electronics. 2014. 29, No 5. P. 2155-2163.

2. Chowdhury S., Swenson B.L., Wong M.H. and Mishra U.K. Current status and scope of gallium nitride-based vertical transistors for high-power electronics application. Semicond. Sci. Technol. 2013. 28, No 7. P. 074014.

3. Avrutin V., Hafiz S.A., Zhang F., Özgür Ü., Morkoc H. and Matulionis A. InGaN light-emitting diodes: Efficiency-limiting processes at high injection. J. Vac. Sci. Technol. A. 2013. 31, No 5. P. 050809.

4. Lu N. and Ferguson I. III-nitrides for energy production: photovoltaic and thermoelectric applications. Semicond. Sci. Technol. 2013. 28, No 7. P. 074023.

5. Beeler M., Trichas E. and Monroy E. III-nitride semiconductors for intersubband optoelectronics: a review. Semicond. Sci. Technol. 2013. 28, No 7. P. 074022.

6. Sydoruk V.A., Zadorozhnyi I., Hardtdegen H., Luth H., Petrychuk M.V., Naumov A.V., Korotyeyev V.V., Kochelap V.A., Belyaev A.E. and Vitusevich S.A. Electronic edge-state and space-charge phenomena in long $\mathrm{GaN}$ nanowires and nanoribbons. Nanotechnology. 2017. 28. P. 135204.

7. Ahi K. Review of GaN-based devices for terahertz operation. Opt. Eng. 2017. 56, No 9. P.090901.
8. Korotyeyev V.V., Kochelap V.A., Kim K.W., and Woolard D.L. Streaming distribution of twodimensional electrons in III-N heterostructures for electrically pumped terahertz generation. Appl. Phys. Lett. 2003. 82, No 16. P. 2643-2645.

9. Kim K.W., Korotyeyev V.V., Kochelap V.A., Klimov A.A., and Woolard D.L. Tunable terahertzfrequency resonances and negative dynamic conductivity of two-dimensional electrons in groupIII nitrides. J. Appl. Phys. 2004. 96, No 11. P. 6488-6491.

10. Knap W., Kachorovskii V., Deng Y., Rumyantsev S., Lu J.-Q., Gaska R., Shur M.S., Simin G., Hu X., Khan M.A., Saylor C.A. and Brunel L.C. Nonresonant detection of terahertz radiation in field effect transistors. J. Appl. Phys. 2002. 91, No 11. P. 9346-9353.

11. Laurent T., Sharma R., Torres J. et al. Voltagecontrolled sub-terahertz radiation transmission through GaN quantum well structure Appl. Phys. Lett. 2011. 99. P. 082101.

12. Santoruvo G., Allain A., Ovchinnikov D. and Matioli E. Magneto-ballistic transport in $\mathrm{GaN}$ nanowires. Appl. Phys. Lett. 2016. 109. P.103102.

13. Bouguen L., Contreras S., Jouault A.B., Konczewicz L., Camassel J., Cordier Y., Azize M., Chenot $\mathrm{S}$. and Baron $\mathrm{N}$. Investigation of $\mathrm{AlGaN} / \mathrm{AlN} / \mathrm{GaN}$ heterostructures for magnetic sensor application from liquid helium temperature to $300{ }^{\circ}$ C. Appl. Phys. Lett. 2008. 92. P. 043504.

14. Gilbertson A.M., Benstock D., Fearn M., Kormanyos A., Ladak S., Emeny M.T., Lambert C.J., Ashley T., Solin S.A. and Cohen L.F. Sub$100-n m$ negative bend resistance ballistic sensors for high spatial resolution magnetic field detection. Appl. Phys. Lett. 2011. 98, No 6. P. 062106.

15. Look D.C. and Sizelove J.R. Predicted maximum mobility in bulk GaN. Appl. Phys. Lett. 2001. 79, No 8. P.1133-1135.

16. Starikov E., Shiktorov P., Gruzinskis V., Varani L., Palermo C., Millithaler J.F. and Regiani L. Frequency limits of terahertz radiation generated by optical-phonon transit-time resonance in quantum wells and heterolayers. Phys. Rev. B. 2007. 76, No 4. P. 045333; Terahertz generation in nitrides due to transit-time resonance assisted by optical phonon emission. J. Phys.: Condens. Matter. 2008. 20, No 38. P. 384209.

17. Lu J.T. and Cao J.C. Monte Carlo study of terahertz generation from streaming distribution of twodimensional electrons in a $\mathrm{GaN}$ quantum well. Semicond. Sci. Technol. 2005. 20, No 8. P. 829833.

18. Barry E.A., Kim K.W., and Kochelap V.A. Hot electrons in group-III nitrides at moderate electric fields. Appl. Phys. Lett. 2002. 80, No 13. P. 23172319. 
19. Yilmazoglu O., Mutamba K., Pavlidis D. and Karaduman T. Measured negative differential resistivity for GaN Gunn diodes on $\mathrm{GaN}$ substrate. Electronics Lett. 2007. 43, No 8. P. 480-482.

20. Syngayivska G.I. and Korotyeyev V.V. Electrical and high-frequency properties of compensated $\mathrm{GaN}$ under electron streaming conditions. Ukr. J. Phys. 2013. 58, No 1. P. 40-55.

21. Korotyeyev V.V. Peculiarities of THzelectromagnetic wave transmission through the $\mathrm{GaN}$ films under conditions of cyclotron and optical phonon transit-time resonances. Semiconductor Physics, Quantum Electronics \& Optoelectronics. 2013. 16, No 1. P. 18-26.

22. Syngayivska G.I., Korotyeyev V.V., Kochelap V.A. and Varani L. Magneto transport in crossed electric and magnetic fields in compensated bulk GaN. $J$. Appl. Phys. 2016. 120. P. 095704.

23. Syngayivska G.I. and Korotyeyev V.V. Electron transport in crossed electric and magnetic fields under the condition of the electron streaming in GaN. Semiconductor Physics, Quantum Electronics \& Optoelectronics. 2015. 18, No 1. P. 79-85.

24. Mitin V.V., Kochelap V.A., Stroscio M.A. Quantum Heterostructures: Microelectronics and Optoelectronics. New York: Cambridge University Press, 1999.

25. Nag B.R. Diffusion equation for hot electron. Phys. Rev. B. 1975. 11, No 8. P. 3031-3036.

26. Gupta M.S. Random walk calculation of diffusion coefficient in two-valley semiconductors. J. Appl. Phys. 1978. 49, No 5. P. 2837-2844.

27. Fauquembergue R., Zimmermann J., Kaszynski A., Constant E. and Microondes G. Diffusion and the power spectral density and correlation function of velocity fluctuation for electrons in $\mathrm{Si}$ and $\mathrm{GaAs}$ by Monte Carlo methods. J. Appl. Phys. 1980. 51, No 2. P. 1065-1071.

28. Ferry D.K. and Barker J.R. Generalized diffusion, mobility, and the velocity autocorrelation function for high-field transport in semiconductors. J. Appl. Phys. 1981. 52, No 2. P. 818-824.

29. Hot Electron Diffusion. Ed. J. Pozhela. Vilnius, Mokslas, 1981 (in Russian).

30. Pozhela J.K and Repshas K.K. Thermoelectric force of hot carriers. phys. status solidi. 1968. 27, No 2. P. 757-762.

31. Ruch J.G. and Kino G.S. Transport properties of GaAs. Phys. Rev. 1968. 174, No 3. P. 921-931.

32. Nougier J.P., Comallonga J. and Rolland M. Pulsed technique for noise temperature measurement. $J$. Phys. E: Sci. Instrum. 1974. 7. P. 287-290.

33. Eichler H.J., Gunter P., Pohl D.W. Laser-Induced Dynamic Grattings. Berlin: Springer-Verlag, Berlin Heidelberg, 1986.

34. Linnros J. and Grivickas V. Carrier-diffusion measurements in silicon with a Fourier-transient- grating method. Phys. Rev. B. 1994. 50. P.1694316955.

35. Starikov E., Shiktorov P., Gruzinskis V., Reggiani L., Varani L., Vaissiere J.C. and Palermo C. Monte Carlo calculations of hot-electron transport and diffusion noise in $\mathrm{GaN}$ and InN. Semicond. Sci. Technol. 2005. 20, No 3. P. 279-285.

36. Wang S., Liu H., Gao B. and Cai H. Monte Carlo calculation of electron diffusion coefficient in wurtzite indium nitride. Appl. Phys. Lett. 2012. 100. P. 142105.

37. Aleksiejūnas R., Podlipskas Ž., Nargelas S., Kadys A., Kolenda M., Nomeika K., Mickevičius J. and Tamulaitis G. Direct Auger recombination and density-dependent hole diffusion in InN. Sci. Reports. 2018. 8. P. 4621.

38. Syngayivska G.I., Korotyeyev V.V. and Kochelap V.A. High-frequency response of $\mathrm{GaN}$ in moderate electric and magnetic fields: interplay between cyclotron and optical phonon transient time resonances. Semicond. Sci. Technol. 2013. 28, No 3. P. 035007.

39. Price P.J. Calculation of hot electron phenomena. Solid-State Electronics. 1978. 21. P. 9-16.

40. Jacoboni C. and Reggiani L. The Monte Carlo method for the solution of charge transport in semiconductors with applications to covalent materials. Rev. Mod. Phys. 1983. 55, No 3. P. 645705.

41. Vosilius I.I. and Levinson I.B. Optical phonon production and galvanomagnetic effects for a largeanisotropy electron distribution. Soviet Physics JETP. 1966. 23, No 6, P. 1104-1107; Galvanomagnetic effects in strong electric fields during nonelastic electron scattering. Soviet Physics JETP. 1967. 25, No 4. P. 672-679.

42. Kochelap V.A., Korotyeyev V.V., Syngayivska G.I. and Varani L. High-field electron transport in $\mathrm{GaN}$ under crossed electric and magnetic fields. J. Phys.: Conf. Series. 2015. 647. P. 012050.

\section{Authors and CV}

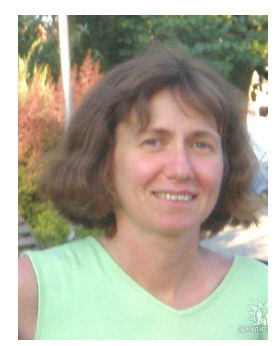

Dr. Galina Syngayivska born 1969 in Drohobych (Lviv region, Ukraine), graduated in physics in 1993, the $\mathrm{Ph} . \mathrm{D}$. degree in solid state physics in 2015 from the V. Lashkaryov Institute of Semiconductor Physics NAS of Ukraine. Since 2016, she is the researcher at the Department of Theoretical Physics in the V. Lashkaryov Institute of Semiconductor Physics, NASU. She is the author of more than 20 publications. Her main research activity is in the field of Monte Carlo simulation of electron transport in nanoscale structures and devices.

E-mail: singg@ukr.net 


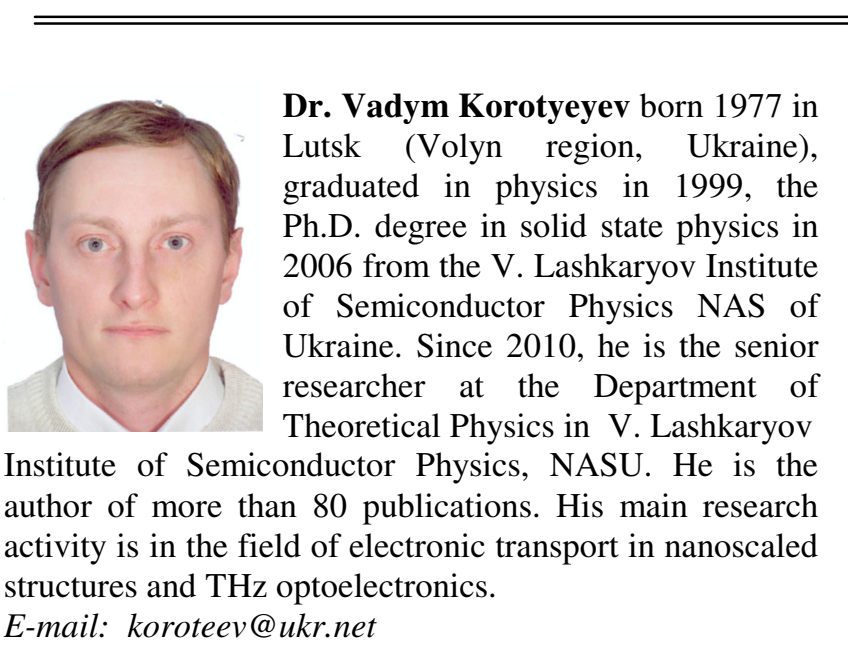

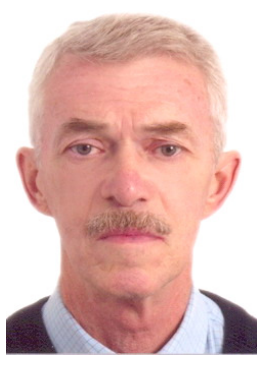

Prof., Dr. Viacheslav A. Kochelap born 1944 in Kyiv (Ukraine), graduated in theoretical physics in 1966 (Kiev State University), the $\mathrm{Ph} . \mathrm{D}$. degree in solid state physics in 1970 from the Institute of Semiconductor Physics NAS of Ukraine. Since 1987, he is full Professor at the Department of Theoretical Physics in the V. Lashkaryov Institute of Semiconductor Physics, NASU. He is the author of more than 250 publications. His main research activity is in the field of electronic transport, fluctuation phenomena and THz-physics of semiconductors and semiconductor nanoscale devices. 